\title{
Theoretical Bounds and System Design for Multipinhole SPECT
}

\author{
Peter Nillius*, Member, IEEE, and Mats Danielsson
}

\begin{abstract}
The pinhole camera in single photon emission computed tomography (SPECT) has an inherent trade-off between resolution and sensitivity. Recent systems overcome this to some extent by utilizing multiple pinholes distributed around the imaging object. The present work is a theoretical study on how to optimally construct such systems. We use an analytic model to analyze the multipinhole SPECT geometry and identify the underlying tradeoffs. One of the results is the derivation of the upper bound for the sensitivity, given the geometric resolution and field-of-view (FOV). Reaching this bound requires an infinitely large detector. However, a sensitivity very close to the upper bound can be achieved by a system with realistic proportions. We show that it is usually possible to get a sensitivity that is $\mathbf{9 5 \% - 9 9 \%}$ of the upper bound. Further analysis reveals a trade-off between sensitivity, magnification, and the number of pinholes. Based on this new theory, we develop a strategy for multipinhole SPECT design, from which a number of example systems are computed. Penetration in the pinhole knife edge is accounted for by using the resolution and sensitivity equivalent apertures.
\end{abstract}

Index Terms-Multipinhole collimator, single photon emission computed tomography (SPECT), small-animal imaging, system analysis and design.

\section{INTRODUCTION}

$\mathbf{I}$ $\mathrm{N}$ VIVO imaging of molecular mechanisms using single photon emission computed tomography (SPECT) and positron emission tomography (PET) is increasingly important in preclinical imaging. This has created the need for systems with higher resolution, especially in small animal imaging. PET resolution is ultimately limited by the range of positrons and the noncolinearity of the emitted photons. SPECT does not have this limitation but here higher resolution is traded for lower sensitivity.

Pinhole collimators have been used successfully to build systems with a high resolution, as a high magnification can be used to overcome the limiting low intrinsic resolution of conventional detectors [1]-[3].

High resolution requires a small pinhole aperture, which drastically reduces the system sensitivity. To counter this, a number

Manuscript received February 22, 2010; accepted March 23, 2010. First published April 08, 2010; current version published June 30, 2010. Asterisk indicates corresponding author.

*P. Nillius is with the Department of Physics, Royal Institute of Technology (KTH), Albanova University Center, SE-106 91 Stockholm, Sweden (e-mail: nillius@mi.physics.kth.se).

M. Danielsson is with the Department of Physics, Royal Institute of Technology (KTH), Albanova University Center, SE-106 91 Stockholm, Sweden (e-mail: md@mi.physics.kth.se).

Color versions of one or more of the figures in this paper are available online at http://ieeexplore.ieee.org.

Digital Object Identifier 10.1109/TMI.2010.2047113 of systems have been built that utilize multiple pinholes, essentially increasing the system sensitivity by a factor equal to the number of pinholes [4]-[14]. Even more ambitious systems using high-resolution detectors have been suggested [15]-[17].

Multipinhole systems have successfully achieved higher resolution and sensitivity. But it is not yet fully understood how to optimally design such systems and what the limits are of this approach. Previous multipinhole SPECT studies are mainly based on simulations and have optimized specific parameters such as the number of pinholes [18] or the properties of the pinholes [19]. The work most similar to ours uses an analytic model to numerically optimize a subset of the parameters, while leaving others fixed, [20].

In the present work, parts of which first appeared in [21], we use a similar analytic model and provide a theoretical analysis of some of the fundamental properties of multipinhole SPECT design. In particular, we derive the theoretical upper bound for the sensitivity given the system resolution and field-of-view (FOV), when the images do not overlap on the detector. Expressing the formulas in terms of the upper bound greatly simplifies the relations, making it easier to understand the underlying properties and trade-offs in multipinhole SPECT design.

The paper is organized as follows. Section II presents the parameters and notation used in the paper as well as some relations associated with multipinhole SPECT. In Section III we derive the sensitivity upper bound. Section IV discusses system optimization and describes our proposed strategy for multipinhole SPECT design. Using this strategy a number of example systems have been computed. These are presented in Section V. Section VI concludes the paper.

\section{METHOD}

We consider a multipinhole SPECT system with $n$ pinhole cameras all focusing on the same spherical FOV for maximum sensitivity. We would like to solve the following problem: To achieve a desired resolution and FOV, how many pinholes cameras should we use and how should they be arranged in order to maximize the system sensitivity? The constraint is that the images should fit on the detector without overlap.

As shown in Fig. 1(a), each camera is characterized by the distance from the center of the FOV to the pinhole $l$, the distance from the FOV to the detector $L$, and the pinhole aperture $a$. The detector could also be slanted relative to its optical axis, however we show that to maximize the sensitivity the detector should be orthogonal to the optical axis as drawn in Fig. 1(a). The proof of this can be found in Appendix A.

As a figure of merit we look at the system resolution and sensitivity at the center of the FOV. While there are variations in 


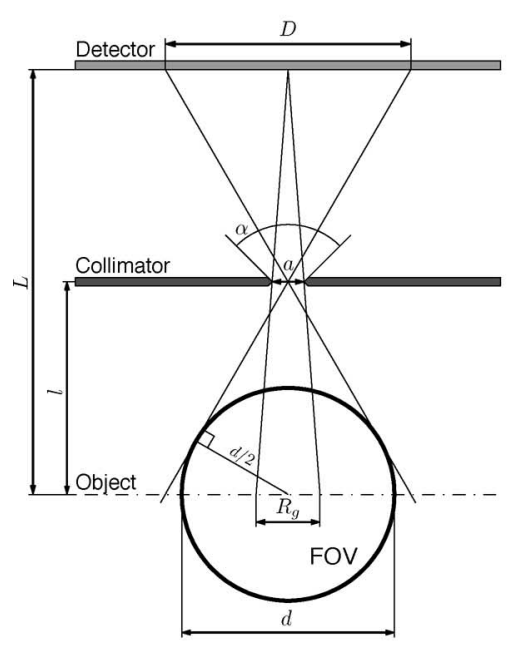

(a)

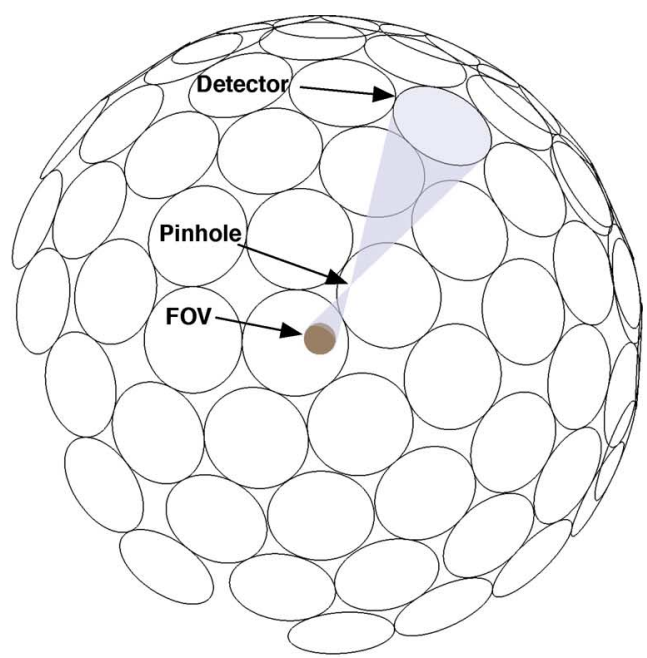

(b)

Fig. 1. Multipinhole camera geometry. (a) Each pinhole camera is characterized by the distance from the center of the FOV to the pinhole $l$, the distance from the FOV to the detector $L$, the pinhole aperture $a$, and the pinhole opening angle $\alpha$. The geometric resolution, $R_{g}$, can be visualized as the width of the cone from a point on the detector through the pinhole. A FOV of diameter $d$ projects an image of diameter $D$ onto the detector. (b) Multiple pinholes project nonoverlapping images onto the detector sphere.

the sensitivity across the FOV, these are very small for systems with evenly distributed pinholes. The sensitivity for a single pinhole decreases with an increasing angle of incidence, but in a multipinhole system this is compensated for by other pinholes lying closer to the radiating point. The variations decrease with an increasing number of pinholes. Also, systems with the collimator close to the FOV have higher variations than when the collimator is further away. For example, our numerical computations, using a FOV of $10 \mathrm{~mm}$, show that a system with 24 pinholes and with the collimator $13 \mathrm{~mm}$ away from the center of the FOV, have $0.7 \%$ variation in the sensitivity over the FOV; 124 pinholes and the same collimator distance result in a sensitivity variation of $0.3 \%$. As the number of pinholes grows to infinity the sensitivity becomes constant over the FOV. We show this analytically in Appendix B where details on the numerical computations can be found as well.

Systems where the pinholes are not evenly distributed, such as systems with cylindrical geometry, will have a drop in sensitivity in the part of the FOV with fewer facing pinholes. This drop depends on the opening angle ${ }^{1}$ of the cylinder. For a cylinder with an opening angle of $25^{\circ}$ and if the collimator is at a reasonable distance from the FOV, the sensitivity variations are relatively small (see Appendix B for details).

When attenuation in the imaging volume is taken into account, the sensitivity at the center is likely to be lower than at the periphery. The sensitivity of peripheral points comes to a higher degree from nearby pinholes, thus attenuation has less of an impact on these points as opposed to points at the center.

The resolution also varies across the FOV, but actually improves towards the periphery as the closer pinholes, which have a higher sensitivity, also give a finer resolution.

\footnotetext{
${ }^{1}$ The opening angle is the angle between the symmetry axis and the line from the center to the edge of the cylinder
}

The system resolution is computed as

$$
R_{t}=\sqrt{R_{g}^{2}+\frac{R_{i}^{2}}{m^{2}}}
$$

where $R_{g}$ is the geometric resolution, $R_{i}$ the intrinsic resolution of the detector, and $m$ is the magnification of the system given by

$$
m=\frac{L-l}{l} .
$$

The geometric resolution, $R_{g}$ is defined to be the width of the beam at the detector from a point source in the center of the FOV, divided by the magnification. This is the same as the width of the FOV for a single point on the detector as plotted in Fig. 1(a). The geometric resolution and the pinhole aperture are related via

$$
R_{g}=a \frac{L}{L-l}
$$

and hence

$$
a=R_{g} \frac{L-l}{L} .
$$

The sensitivity, at the center of the FOV, for a single pinhole is the ratio of the pinhole area to the area of the collimator sphere. Approximating the spherical cap area of the pinhole with the area of a disc, we get the following expression for the single pinhole sensitivity:

$$
S_{\text {single }}=\frac{\pi a^{2}}{16 \pi l^{2}}=\frac{a^{2}}{16 l^{2}} .
$$

Replacing the aperture with the geometric resolution by inserting (4) in (5) results in

$$
S_{\text {single }}=\frac{R_{g}^{2}(L-l)^{2}}{16 l^{2} L^{2}} .
$$


This equation illustrates the resolution-sensitivity trade-off of the pinhole camera. A reduction in the resolution creates the same reduction squared in the sensitivity.

The sensitivity for a system with $n$ pinholes is

$$
S=n S_{\text {single }} .
$$

A spherical FOV of diameter $d$ projects a circular image onto the detectors. The image diameter, $D$, can be derived from the proportional triangles, below and above the pinhole, in Fig. 1(a), resulting in

$$
D=d \frac{L-l}{\sqrt{l^{2}-\frac{d^{2}}{4}}} .
$$

The images should not overlap on the detector sphere. Again, approximating the subtended spherical cap area with area of the image disc, this results in the following inequality:

$$
n \pi \frac{D^{2}}{4} \leq \rho 4 \pi L^{2}
$$

where $\rho$ is the best packing density of equal circles on a sphere. The best packing density varies with the number of circles, i.e., pinholes. For 20-90 circles $\rho$ lies between 0.81 and 0.86 [22]. As the number of circles grows the density converges to the best packing density of equal circles on $\mathcal{R}^{2}$, which is $\pi / \sqrt{12} \approx$ 0.9069 , but a more realistic and slightly conservative estimate for $\rho$ is 0.84 , which is the value used throughout the paper.

\section{A. Pinhole Knife Penetration Effects}

A knife edge pinhole with opening angle $\alpha$ will have some photons penetrating the knife edge, in effect giving a higher sensitivity. This can be accounted for by using a larger aperture with no penetration effects resulting in the equivalent sensitivity as with penetration. The sensitivity equivalent aperture diameter in the center of the FOV as derived in [23] is given by

$$
a_{\mathrm{se}}=\sqrt{a^{2}+\frac{2}{\mu} a \tan \frac{\alpha}{2}+\frac{2}{\mu^{2}} \tan ^{2} \frac{\alpha}{2}}
$$

where $\mu$ is the linear attenuation coefficient for the knife edge material. The sensitivity, including penetration effects, for a single pinhole is

$$
S_{p, \text { single }}=\frac{a_{\mathrm{se}}^{2}}{16 l^{2}}
$$

and for the whole system

$$
S_{p}=n S_{p, \text { single }}
$$

The resolution equivalent aperture can, in the same manner, be used to take into account the penetration effects on the resolution. The resolution equivalent aperture diameter in terms of the FWHM is given by [24] and can be computed as

$$
a_{\mathrm{re}}=a+\frac{\ln 2}{\mu} \tan \frac{\alpha}{2}
$$

hence the geometric resolution in this case becomes

$$
R_{g, p}=a_{\mathrm{re}} \frac{L}{L-l} .
$$

We use the penetration formulas for computing and evaluating our example systems, but do not include them in the optimization. The reason is twofold. First of all, bringing in the penetration formulas renders the analysis impossible. We would have to fall back to numerical optimization, which would not give as many insights into the underlying properties of multipinhole SPECT geometry.

The second reason is that the resolution and sensitivity equivalent apertures affect the optimization in a peculiar way. The $a_{\mathrm{se}}$ is larger than $a_{\mathrm{re}}$, which results in a higher sensitivity at an equivalent resolution. This difference increases with 1) a larger pinhole opening angle and 2) a smaller aperture, thus promoting systems with those properties. However, the added sensitivity lies in the tail of the geometric point spread function and it is nontrivial how this added sensitivity will contribute to the quality of the reconstructed image. One would have to look at the reconstructed image to faithfully evaluate this, which is beyond the scope of this paper.

With the sensitivity equivalent aperture, the drop in sensitivity at the edge of the FOV is larger than without penetration effects. When evaluating the example systems, we also compute the sensitivity at the edge of the FOV, using the full, off-center, formula for the sensitivity including penetration, according to [23].

\section{Derivation of the Sensitivity UpPer Bound}

What multipinhole SPECT system, according to the model presented in the previous section, maximizes the sensitivity given the resolution and FOV size? As it turns out, that system is infinitely large. While such a system is purely hypothetical and cannot be built, it defines the upper bound of the sensitivity, that is achievable, in the context of our model. This section derives this upper bound.

Combining (8) and (9) and isolating $n$ leads to

$$
n \leq \rho \frac{16 L^{2}\left(l^{2}-\frac{d^{2}}{4}\right)}{d^{2}(L-l)^{2}} .
$$

To maximize the sensitivity, we use the maximum number of pinholes according to (15) such that

$$
n=\rho \frac{16 L^{2}\left(l^{2}-\frac{d^{2}}{4}\right)}{d^{2}(L-l)^{2}} .
$$

Combining (6), (7), and (16) and after some algebraic manipulation we obtain

$$
\begin{aligned}
S & =\rho \frac{16 L^{2}\left(l^{2}-\frac{d^{2}}{4}\right)}{d^{2}(L-l)^{2}} \frac{R_{g}^{2}(L-l)^{2}}{16 l^{2} L^{2}} \\
& =\rho \frac{R_{g}^{2}\left(1-\frac{d^{2}}{4 l^{2}}\right)}{d^{2}} .
\end{aligned}
$$


From the expression in (17) we see that for a fixed geometric resolution, $R_{g}$, and FOV, $d$, the sensitivity is maximized when the distance to the collimator, $l$, is as large as possible and that

$$
S \rightarrow \rho \frac{R_{g}^{2}}{d^{2}} \text { when } l \rightarrow \infty
$$

which also means that $L \rightarrow \infty$. Hence the upper bound, or more precisely the supremum of the sensitivity is

$$
S_{\text {sup }}=\sup _{L>l>0} S=\rho \frac{R_{g}^{2}}{d^{2}} .
$$

What is happening when $L$ grows is that both the area of the detector sphere and the total area of the images grow. The image area grows faster resulting in a decreasing number of pinholes. At the same time, the sensitivity of each pinhole increases because the aperture of each pinhole can be increased without loss in resolution. These changes in $n$ and $S_{\text {single }}$ cancel out such that the total sensitivity becomes independent of $L$.

When the collimator position $l$ grows and $L$ is fixed, the number of pinholes is increased. This is because the magnification of the system is reduced so that more images fit onto the detector. At the same time the single pinhole sensitivity decreases as the aperture has to be reduced to maintain the resolution. These effects do not cancel out; the number of pinholes increases faster such that the total sensitivity is increased as $l$ is increased.

Of course, the sensitivity does not grow to infinity but towards an asymptotic value which is the upper bound in (19). While an infinitely large system is purely hypothetical, the upper bound plays an important roll in the subsequent sections. Also, note that the sensitivity comes close the upper bound at very reasonable proportions. For example, already at $l=2 d$ the sensitivity is $94 \%$ of the upper bound.

Fig. 2 shows the upper bound for various geometric resolutions and FOVs. For example, with a resolution of $1 \mathrm{~mm}$ it is possible to get a sensitivity of $0.5 \%-1 \%$, if the FOV is small enough. To be able to achieve a resolution of $0.1 \mathrm{~mm}$ we need to go down to a $9 \mathrm{~mm}$ FOV for a maximum sensitivity of $0.01 \%$.

\section{A. Upper Bound in Terms of System Resolution}

The upper bound in the previous section involves the geometric resolution. To express it in terms of the system resolution we use (1) to write $R_{g}$ in terms of $R_{t}$ and $R_{i}$, and plug it into (17), resulting in

$$
S=\rho \frac{\left(R_{t}^{2}-\frac{R_{i}^{2}}{m^{2}}\right)\left(1-\frac{d^{2}}{4 l^{2}}\right)}{d^{2}} .
$$

This expression is maximized when $l \rightarrow \infty$ and hence $L \rightarrow \infty$. By also letting $m$ grow enough, i.e., $L$ grows faster than $l$, we can get arbitrary close to

$$
S_{\mathrm{sup}, R_{t}}=\rho \frac{R_{t}^{2}}{d^{2}}
$$

which is the upper bound for the system sensitivity given the system resolution and the FOV.

This upper bound is identical to the previous one based on the geometric resolution, but to get close to this upper bound

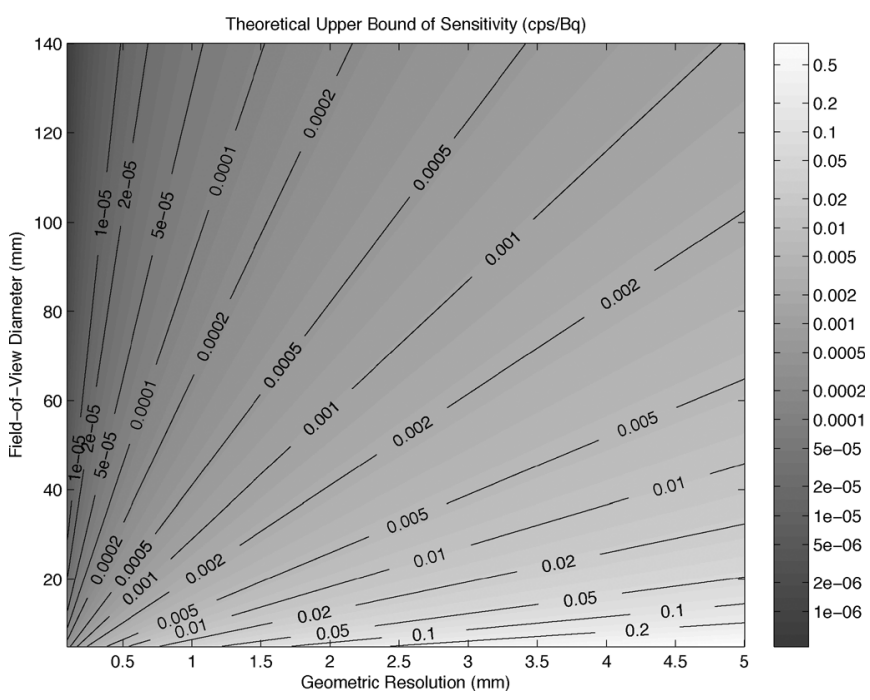

Fig. 2. Theoretical upper bound of the sensitivity for multipinholes SPECT systems with nonoverlapping images. The gray value and contours indicate the sensitivity upper bound for different geometric resolutions and FOVs. As it usually is possible to design systems with sensitivities up to $99 \%$ of the upper bound, this plot illustrates the resolution-sensitivity-FOV trade-off in a nutshell.

the intrinsic resolution must be several times smaller than the magnified geometric resolution. To see the effect when this is not the case, we introduce the rate of oversampling, $k$, to denote the ratio of the magnified geometric resolution to the intrinsic resolution, defined as

$$
k=\frac{m R_{g}}{R_{i}} .
$$

In combination with (1) we obtain

$$
R_{g}=\frac{R_{t}}{\sqrt{1+\frac{1}{k^{2}}}} .
$$

Inserting this expression in (19), we find that, for a fixed rate of oversampling, the upper bound of the sensitivity is reduced with a factor of $1 /\left(1+1 / k^{2}\right)$ resulting in

$$
S_{\mathrm{sup}, R_{t}, k}=\rho \frac{R_{t}^{2}}{d^{2}} \frac{1}{1+\frac{1}{k^{2}}} .
$$

For example, a rate of oversampling of $k=1$ reduces the maximum sensitivity with a factor of $0.5, k=2$ with a factor of 0.8 , $k=3$ with a factor of 0.9 , and so on.

Another study, [25], concurrent with ours, derives the maximum sensitivity while incorporating the effects of penetration in the pinhole knife edge, in the context of brain SPECT. Through an approximation using a first-order Taylor expansion they identify two regimes, one for low-resolution and one for high-resolution detectors. For low-resolution detectors they arrive at the same upper bound as in (21). For high-resolution detectors, they find an increase in the maximum sensitivity by a factor of $2 /\left(\mu R_{i}+(\ln 2)^{2}\right)$. This increase can be ascribed to the penetration effects, which appear beneficial for large pinhole opening angles and small pinhole apertures, as discussed in Section II-A. Large pinhole opening angles occur in systems with the collimator close to the FOV. A small pinhole aperture is needed to maintain resolution when the detector is close to 
the collimator, which require a detector of high intrinsic resolution, due to the minifaction in such systems. The maximum sensitivity is, in this case, reached when the pinhole aperture is zero such that the pinhole consists only of its knife edge.

\section{B. Other Detector Geometries}

The upper bound is derived for systems with detectors that are orthogonal to the optical axis, as in the case of the sphere. Since this configuration is optimal, in the sense that is maximizes the sensitivity per solid angle, the upper bound holds for all other geometries. So, although it is not as tight for other geometries it still bounds the sensitivity given the resolution and FOV size.

\section{EQuivalent Configurations AND System Design}

Because of the multipinhole geometry, we cannot optimize the system parameters to maximize the sensitivity without constraints, as the system size will grow to infinity. Limiting the system size will not help either as then the number of pinholes will grow to infinity. A deeper understanding of the underlying properties of multipinhole SPECT geometry is needed. In this section we derive new formulas and relations between the various system properties, that will be of great help when doing the necessary trade-offs in multipinhole SPECT design.

For a given sensitivity below the upper bound, there are many configurations with equivalent performance. These configurations correspond to different values in the number of pinholes, the pinhole diameters and the placement of the detector layer. As we will see, this gives us flexibility to select some of the parameters as we please when designing our system, such as the number of pinholes and the magnification of the system.

In the previous section we saw that the sensitivity and the sensitivity upper bound differed by a factor depending only on $l$ and $d$. Define the ratio between the sensitivity and the sensitivity upper bound as

$$
r=\frac{S}{S_{\text {sup }}} .
$$

Inserting (17) and (19) into (25) results in

$$
r=1-\frac{d^{2}}{4 l^{2}} \text {. }
$$

Solving for $l$ leads to

$$
l=\frac{d}{2} \frac{1}{\sqrt{1-r}} .
$$

Consequently, the position of the pinhole collimator is directly linked to the sensitivity of the system. For example, $S=0.5 S_{\text {sup }}$ puts the collimator at $l=(d) /(\sqrt{2})$, $S=0.99 S_{\text {sup }}$ at $l=5 d$ and of course $S=S_{\text {sup }}$ will put the collimator at infinity. Consider a system with fixed geometric resolution, $R_{g}$ and FOV diameter, $d$. The sensitivity upper bound for this system is given by (19). Choosing a desired system sensitivity then defines the sensitivity to upper bound ratio, $r$, which in turn sets the position of the collimator, according to (27).

We are now free to position the detector by choosing $L$. Different values of $L$ correspond to systems with equal sensitivity, but with different number of pinholes, $n$, given by (16), and

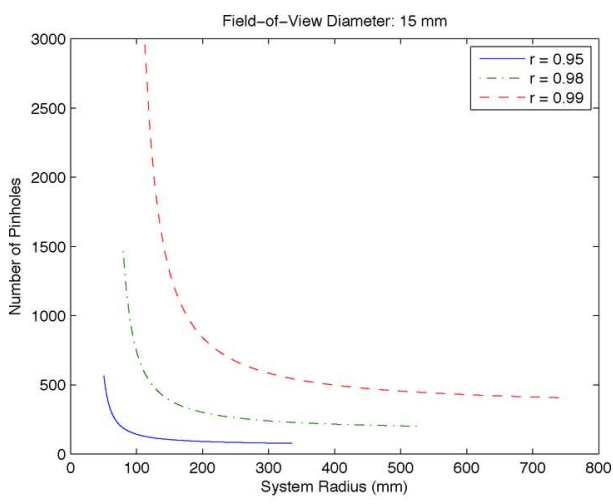

(a)

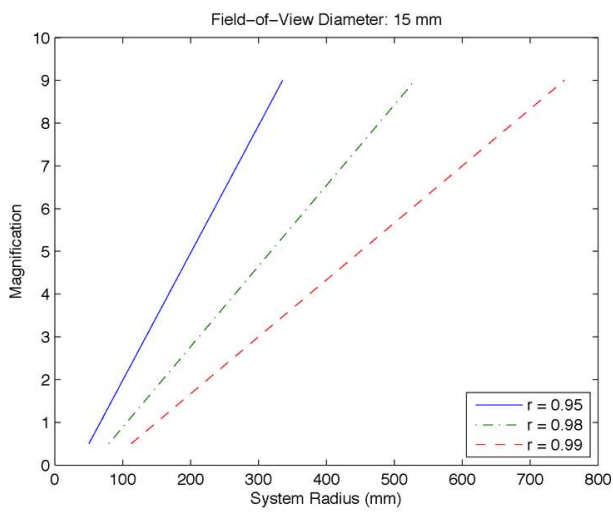

(b)

Fig. 3. For a given resolution, FOV and sensitivity there are several configurations with the same performance. Under these restrictions the system parameters are coupled. For example (a) shows the number of pinholes and (b) the magnification as the system radius changes. Allowing only very small changes in the sensitivity gives further flexibility in the selection of parameters as shown by the lines for sensitivities of $95 \%, 98 \%$, and $99 \%$ of the theoretical upper bound.

system magnification $m$, given by (2). Examples of how $n$ and $m$ vary with $L$, and for various values of $r$, can be seen in Fig. 3 .

As can be seen, even though $r$ is very close to 1 , which means the performance is close the theoretical maximum, the system parameters are within realistic proportions. Fig. 3 also shows that small changes in $r$ have a big impact on the number of pinholes as well as the magnification of the system. This means that we can trade a few percent of the sensitivity to get a greater flexibility in choosing the number of pinholes and the magnification. In fact, $n, m$ and $r$ are related to each other independently of all the other parameters. This can most easily be derived by rewriting (16) such that

$$
n=\rho \frac{16 L^{2} l^{2}\left(1-\frac{d^{2}}{4 l^{2}}\right)}{d^{2}(L-l)^{2}}=\rho \frac{16 L^{2} r}{d^{2} m^{2}}
$$

and using (27) and (2) to eliminate $L$ from (28). Solving for $r$ results in

$$
r=\frac{1}{1+\frac{4 \rho\left(1+\frac{1}{m}\right)^{2}}{n}} .
$$

This expression for $r$ is independent of the resolution and the FOV and only depends on the number of pinholes, $n$ and the magnification $m$. Fig. 4 shows this function as a contour plot. 


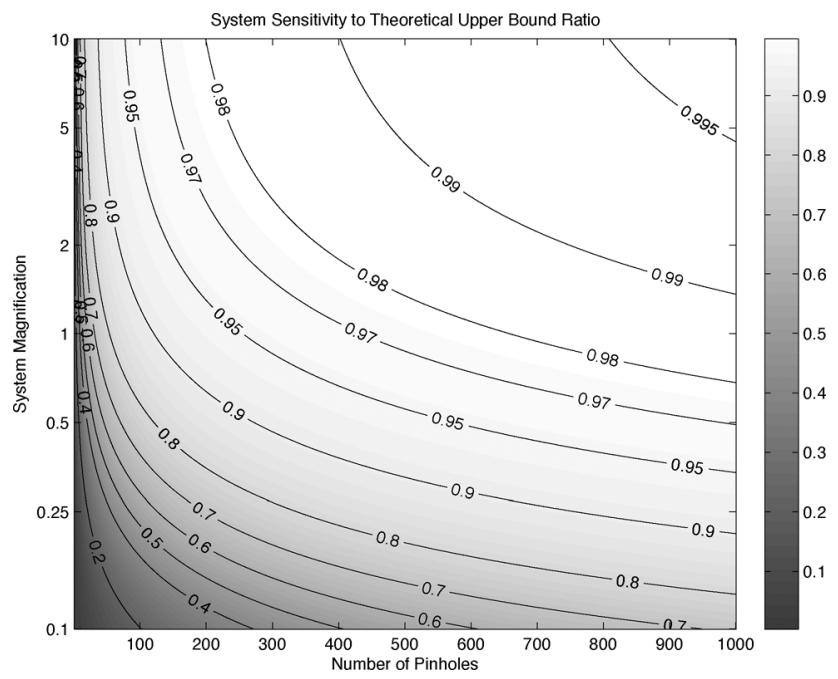

Fig. 4. The sensitivity-to-upper bound ratio $r$ can be expressed as a function depending only on the magnification, $m$ and the number of pinholes, $n$. The gray value and contours indicate the sensitivity-to-upper bound ratio achieved by a system with a particular $n$ and $m$. As can be seen there are many options to design a system which has a sensitivity of over $95 \%$ of its theoretical upper bound. A system with low magnification requires more pinholes than a system with high magnification, to achieve a high sensitivity.

From this figure it can be seen that we usually can achieve a sensitivity that is $95 \%$ up to $99 \%$ of the theoretical upper bound. The more pinholes the higher sensitivity. Also, a system with high magnification requires fewer pinholes to get a high sensitivity, which is interesting since a high magnification often is used in small animal imaging due to the low intrinsic resolution of conventional detectors. For example, a system with magnification $m=4$ requires 99 pinholes to get $95 \%$ of the upper bound sensitivity. A system with minification on the other hand requires more pinholes, e.g., a system with magnification factor $m=0.5$ requires 568 pinholes to get a sensitivity of $95 \%$ of the upper bound. A factor $m=0.25$ require 1577 pinholes (outside the limits of the plot) for the same sensitivity ratio.

A high magnification factor also makes the system large, requiring a large detector area. Fig. 5 shows the system radius divided by the FOV radius - to make it scale invariant - as a function of the number of pinholes and system magnification.

\section{A. System Design}

Using the formulas presented here there are many ways to design a SPECT system. Here we outline one method, which is used in computing the example systems in Section V.

As Fig. 4 shows, it is usually possible to achieve $90 \%-99 \%$ of the theoretical upper bound. This means we can use the simple formula for the upper bound to do the trade-off between resolution, sensitivity and FOV. To be able to choose the total system resolution we need to use the upper bound in (24) which includes the oversampling factor. The oversampling factor is unknown at this point, but if we design a system with a large enough oversampling, this factor is small. Thus, the first step is to choose the system resolution, $R_{t}$, and the FOV diameter, $d$, knowing that the system sensitivity will be slightly lower than the theoretical upper bound, depending on the degree of oversampling.

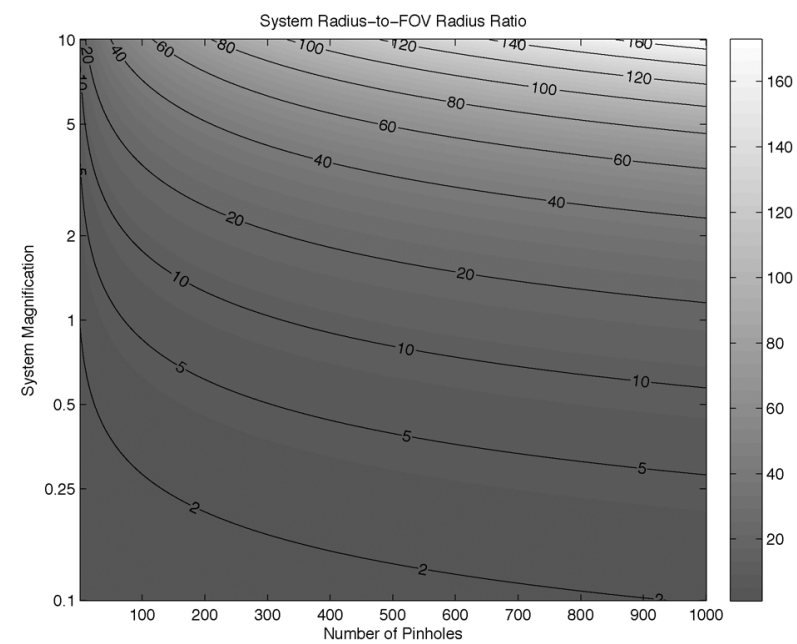

Fig. 5. The detector-to-FOV radius ratio, $L /(d / 2)$ can also be expressed as a function of the number of pinholes, $n$ and the magnification, $m$. For example, for a FOV diameter of $2 \mathrm{dm}$, the plot shows the detector radius in dm's.

The next step is to choose the system magnification $m$ and the number of pinholes $n$, using (29) and Fig. 4 for guidance. Depending on the intrinsic resolution of the detector, the magnification needs to be sufficiently large to ensure that the detector oversamples the geometric resolution. The required magnification, for a given oversampling factor, $k$ can be computed by combining (22) and (23) such that

$$
m=\frac{R_{i} \sqrt{k^{2}+1}}{R_{t}} .
$$

The number of pinholes needed depends on the magnification factor. The minimum number of pinholes required to achieve a certain sensitivity can be found by solving for $n$ in (29) such that

$$
n=\frac{4 \rho\left(1+\frac{1}{m}\right)^{2}}{\frac{1}{r}-1} .
$$

A higher number of pinholes may be chosen as that increases the sensitivity. This is potentially useful for a stationary system, in order to get a sufficient number of projections for image reconstruction. Moreover, some pinhole numbers have especially efficient packings or other desirable symmetries for construction purposes.

Once the system resolution, the FOV, the magnification, the number of pinholes and the sensitivity ratio are set, we can compute all the remaining parameters of the system, namely $l, L$, and $a$.

To account for the pinhole knife edge penetration effects, we compute the geometric resolution as

$$
R_{g, p}=\sqrt{R_{t}^{2}-\frac{R_{i}^{2}}{m^{2}}}
$$

and use it to compute the resolution equivalent aperture

$$
a_{\mathrm{re}}=R_{g, p} \frac{L-l}{L} .
$$


TABLE I

Multipinhole SPECT System Design: STEPS USED to COMPUTE THE EXAMPLE SYSTEMS IN THE PAPER

1) Select the system resolution $R_{t}$ and the field-of-view $d$, by doing the resolution-FOV-sensitivity trade-off using (19) and/or Fig. 2.

2) Do another trade-off and select the magnification $m$, the number of pinholes $n$ and the sensitivity upper bound ratio $r$, using Fig. 4 and/or (29) or (31). The magnification should be sufficiently large for the detector to oversample the geometric resolution.

3) Compute the collimator position, $l$, using (27).

4) Compute the detector position: $L=(m+1) l$.

5) Compute the geometric resolution including penetration using (32).

6) Compute the resolution equivalent pinhole aperture, $a_{r e}$, using (33).

7) Compute the pinhole aperture, $a$, through (34).

The actual aperture is obtained by subtracting the contribution from the penetration effects

$$
a=a_{\mathrm{re}}-\frac{\ln 2}{\mu} \tan \frac{\alpha}{2} .
$$

Table I outlines the procedure in algorithmic form.

\section{EXAMPLE SYSTEMS}

Using the presented theory for multipinhole SPECT design, we have computed and evaluated a number of example systems. These include one set intended for small animal imaging and one set for brain SPECT.

\section{A. Small Animal Spect}

For comparison, our small animal systems have been computed according to the same specifications as some of the systems in [20]. For example, all computations were made for ${ }^{99 m}$ Tc $140 \mathrm{keV}$. Using gold as the pinhole aperture material, this results in an attenuation coefficient of $\mu=4.27 \mathrm{~mm}^{-1}$. The FOV diameter was set to $12 \mathrm{~mm}$.

Two sets of systems were computed. One using conventional NaI scintillation detectors, the other using high-resolution detectors. The intrinsic resolution of the conventional and high-resolution detectors was set to be 3.2 and $0.1 \mathrm{~mm}$, respectively. For each detector type, two systems with system resolution $0.61 \mathrm{~mm}$ and $0.87 \mathrm{~mm}$ were computed, again to compare with [20]. The systems were computed according to the procedure in Table I. The pinhole opening angle is set as small as possible, without obstructing the FOV, resulting in $\alpha=2 \sin ^{-1}(d / 2 l)$.

The low-resolution detectors need a high magnification to compensate for the low intrinsic resolution. We have chosen a magnification of 16, which results in an oversampling factor of 3-4. This also gives great flexibility in selecting the number of pinholes, as it can be either low or very high and still resulting in a high sensitivity. We selected 124 pinholes as this gives enough projections for image reconstruction, with a stationary system. An example of this packing, described in [26], can be seen in Fig. 1(b). This selection of magnification and number of pinholes results in a sensitivity to upper bound ratio of 0.97 .

The first two columns of Table II show the computed systems for the low-resolution detectors. A full covering of the detector sphere is assumed. However, for comparison we have also computed the sensitivity with the same partial covering as used in [20]. This factor is 0.91 and the resulting sensitivity is
TABLE II

Computed Multipinhole Systems for Small Animal SPECT. Two Sets of Systems Computed Using THE Presented Theory, Two BAsed ON LOW-RESOLUTION DETECTORS $\left(R_{i}=3.2 \mathrm{MM}\right)$ AND TWO BASED ON High-RESOLUTION DETECTORS ( $\left.R_{i}=0.1 \mathrm{MM}\right)$. TOTAL RESOLUTION WAS SET TO 0.61 AND 0.87 FOR COMPARISON With [20]. TOTAL SENSITIVITY With the SAME PARTIAL COVERING AS IN [20] IS PRESENTED AT THE BOTTOM OF THE TABLE

\begin{tabular}{lccccc}
\hline Pinhole aperture (mm) & $a$ & 0.51 & 0.77 & 0.16 & 0.25 \\
Pinhole opening angle & $\alpha$ & $20^{\circ}$ & $20^{\circ}$ & $20^{\circ}$ & $20^{\circ}$ \\
Collimator radius (mm) & $l$ & 34.8 & 34.8 & 35 & 35 \\
Detector radius (mm) & $L$ & 592 & 592 & 52.5 & 52.5 \\
Number of pinholes & $n$ & 124 & 124 & 1000 & 1000 \\
Intrinsic Res. (mm) & $R_{i}$ & 3.2 & 3.2 & 0.1 & 0.1 \\
\hline FOV diameter (mm) & $d$ & 12 & 12 & 12 & 12 \\
Magnification & $m$ & 16 & 16 & 0.5 & 0.5 \\
Oversampling & $k$ & 2.9 & 4.2 & 2.9 & 4.2 \\
Packing dens. & $\rho$ & 0.84 & 0.84 & 0.84 & 0.84 \\
Res. equiv. aperture (mm) & $a_{r e}$ & 0.54 & 0.80 & 0.19 & 0.28 \\
Sens. equiv. aperture (mm) & $a_{s e}$ & 0.56 & 0.81 & 0.21 & 0.30 \\
Geom. Res. (mm) & $R_{g}$ & 0.55 & 0.82 & 0.49 & 0.76 \\
Geom. Res. w. penetr. (mm) & $R_{g, p}$, & 0.58 & 0.85 & 0.58 & 0.85 \\
System Resolution (mm) & $R_{t}$ & $\mathbf{0 . 6 1}$ & $\mathbf{0 . 8 7}$ & $\mathbf{0 . 6 1}$ & $\mathbf{0 . 8 7}$ \\
Sensitivity (\%) & $S$ & 0.17 & 0.38 & 0.14 & 0.33 \\
Sensitivity upper bound $(\%)$ & $S_{s u p}$ & 0.17 & 0.39 & 0.14 & 0.34 \\
Sens. to upper bound ratio & $r$ & 0.970 & 0.970 & 0.971 & 0.971 \\
Total Sensitivity (\%) & $S_{p}$ & $\mathbf{0 . 2 0}$ & $\mathbf{0 . 4 2}$ & $\mathbf{0 . 2 2}$ & $\mathbf{0 . 4 5}$ \\
Tot. Sens. at periphery(\%) & $S_{p}(d / 2)$ & 0.18 & 0.40 & 0.18 & 0.39 \\
Total Sens., part. cover. (\%) & $S_{p, p a r t}$ & $\mathbf{0 . 1 8}$ & $\mathbf{0 . 3 8}$ & $\mathbf{0 . 1 9}$ & $\mathbf{0 . 3 9}$ \\
\hline
\end{tabular}

listed at the bottom of the table. The sensitivity, 0.18 and 0.38 , is comparable to that of [20], who reported 0.17 and 0.35 for matching resolution and FOV. Their systems are numerically optimized, with some parameters fixed, such as the collimator position, while our systems are computed analytically from a selected magnification and pinhole number. Consequently, there are several configurations with equal performance, which shows that there is flexibility in how the parameters may be set.

The high-resolution detectors allow us to have low magnification, even below 1. This makes the system small, but also requires more pinholes to get a high sensitivity. We have selected a magnification of 0.5 , giving 3-4 times oversampling, and 1000 pinholes, resulting in a sensitivity to upper bound ratio of 0.97 . The resulting systems are listed in the two last columns of Table II. The total sensitivity is $20 \%$ and $12 \%$ higher than the corresponding low-resolution detector system. However, the reason for this is due to the penetration formulas. The sensitivity, without penetration effects, is in this case, actually lower, 0.14 and 0.33. The high-resolution detectors have smaller pinhole apertures and the smaller the aperture the greater the difference between the resolution equivalent and sensitivity equivalent diameters, as discussed in Section II-A. There is no other reason why a low-resolution detector cannot result in the same performance. The main advantage is that the high-resolution detectors result in a much smaller system.

The sensitivity at the edge of the FOV, denoted as $S_{p}(d / 2)$ is listed second last in Table II. The sensitivity drop at the edge ranges between $5 \%-21 \%$.

\section{B. Brain SPECT}

To further demonstrate the theory, we have computed a set of systems with a larger FOV of $210 \mathrm{~mm}$, suitable for brain SPECT. Four systems have been computed with system resolutions of $1,2,4$, and $8 \mathrm{~mm}$. 
TABLE III

COMPUTED MULTIPINHOLE SPECT SYSTEMS WiTH A FOV OF 210 MM AND RESOLUTIONS OF $1,2,4$, AND 8 MM

\begin{tabular}{lccccc}
\hline Pinhole aperture (mm) & $a$ & 0.52 & 0.81 & 1.1 & 1.3 \\
Pinhole opening angle & $\alpha$ & $39^{\circ}$ & $40^{\circ}$ & $38^{\circ}$ & $38^{\circ}$ \\
Collimator radius (mm) & $l$ & 314 & 305 & 320 & 321 \\
Detector radius (mm) & $L$ & 942 & 610 & 479 & 401 \\
Number of pinholes & $n$ & 60 & 100 & 250 & 700 \\
Intrinsic Res. (mm) & $R_{i}$ & 1 & 1 & 1 & 1 \\
\hline FOV diameter (mm) & $d$ & 210 & 210 & 210 & 210 \\
Magnification & $m$ & 2 & 1 & 0.5 & 0.25 \\
Oversampling & $k$ & 1.7 & 1.7 & 1.7 & 1.7 \\
Packing dens. & $\rho$ & 0.84 & 0.84 & 0.84 & 0.84 \\
Res. equiv. aperture (mm) & $a_{r e}$ & 0.58 & 0.87 & 1.15 & 1.39 \\
Sens. equiv. aperture (mm) & $a_{s e}$ & 0.61 & 0.90 & 1.18 & 1.41 \\
Geom. Res. (mm) & $R_{g}$ & 0.78 & 1.6 & 3.3 & 6.6 \\
Geom. Res. w. penetr. (mm) & $R_{g, p}$ & 0.87 & 1.7 & 3.5 & 6.9 \\
System Resolution (mm) & $R_{t}$ & $\mathbf{1}$ & $\mathbf{2}$ & $\mathbf{4}$ & $\mathbf{8}$ \\
Sensitivity (\%) & $S$ & 0.0010 & 0.0044 & 0.018 & 0.075 \\
Sensitivity upper bound (\%) & $S_{s u p}$ & 0.0012 & 0.0050 & 0.021 & 0.084 \\
Sens. to upper bound ratio & $r$ & 0.888 & 0.882 & 0.892 & 0.893 \\
Total Sensitivity (\%) & $S_{p}$ & $\mathbf{0 . 0 0 1 4}$ & $\mathbf{0 . 0 0 5 4}$ & $\mathbf{0 . 0 2 1}$ & $\mathbf{0 . 0 8 5}$ \\
Tot. Sens. at periphery(\%) & $S_{p}(d / 2)$ & 0.0012 & 0.0049 & 0.020 & 0.080 \\
Total Sens., part. cover. (\%) & $S_{p, p a r t}$ & $\mathbf{0 . 0 0 1 3}$ & $\mathbf{0 . 0 0 4 9}$ & $\mathbf{0 . 0 1 9}$ & $\mathbf{0 . 0 7 7}$ \\
\hline
\end{tabular}

To keep the systems at reasonable sizes, we have settled for a lower sensitivity to upper bound ratio of about 0.89 , as this places the collimator at about $1.5 d$. The magnification is, for the same reason, kept as low as possible. It was chosen to give an oversampling factor of about 1.7. The number of pinholes needed to arrive at the desired $r=0.89$ was estimated using (31).

The parameters, including total sensitivity, are shown in Table III.

\section{SUMMARY AND CONCLUSION}

SPECT systems with multiple pinholes have successfully been used to improve resolution and sensitivity. We use an analytic model and derive a theoretical upper bound beyond which the performance of multipinhole SPECT cannot be pushed, even if the detector is infinitely large. This bound is in fact reached with a detector of infinite size. Moreover, we show that it is usually possible, even with a system of realistic proportions, to get a sensitivity that is up to $99 \%$ of the upper bound, which means that the simple expression for the theoretical upper bound, in fact, captures the resolution-sensitivity-FOV trade-off in multipinhole SPECT.

For a sensitivity below the upper bound there exist several system configurations with the same performance. This gives us flexibility to freely set some of the parameters of the SPECT system. Furthermore, if the sensitivity is allowed to vary a few percent, this gives us further freedom to set the various system parameters. For example, we show how the number of pinholes, the camera magnification and the sensitivity-to-upper bound ratio are coupled, independently of all other variables.

Another interesting result is that it is possible to get the same performance regardless of the intrinsic resolution of the detector. With high-resolution detectors one can use minification to fit more images onto the detector sphere and hence more pinholes. However, the increasing number of pinholes is counteracted by decreasing pinhole apertures, resulting in the same resolution-sensitivity trade-off as for low-resolution detectors. On the other hand, using low-resolution detectors results in much larger systems.

We model penetration effects of the pinhole edge using the resolution and sensitivity equivalent apertures. The equivalence

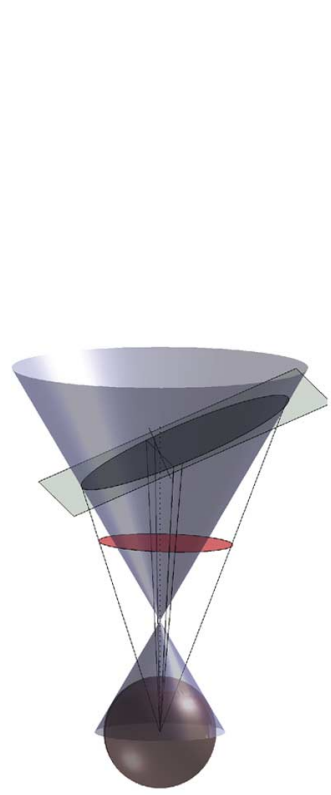

(a)

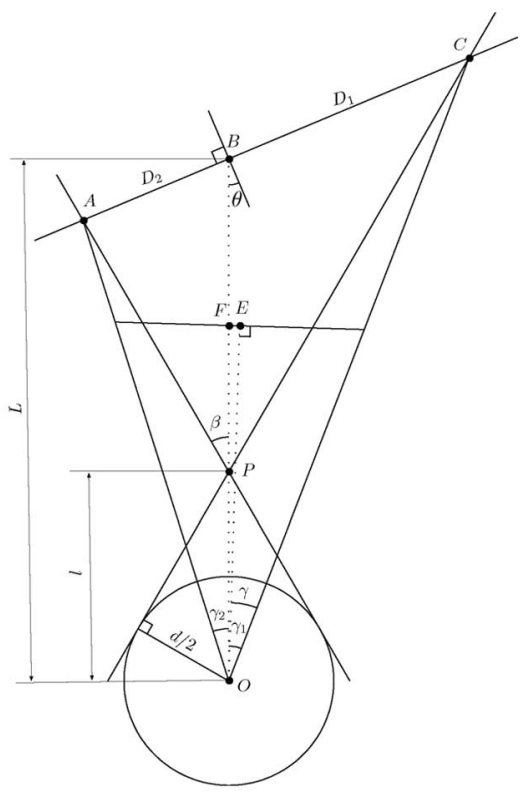

(b)
Fig. 6. Pinhole geometry with a slanted detector. (a) A spherical FOV projects an elliptical image (upper ellipse). This ellipse projects to another ellipse on the tangent plane of the unit sphere (lower ellipse). The solid angle subtended by the image is minimized when the area of projected ellipse is minimized. (b) Crosssection along the ellipses major axes. The major radius of the projected ellipse is $\tan \gamma$ where $\gamma$ can be computed as the average of the two side angles $\gamma_{1}$ and $\gamma_{2}$.

formulas have a peculiar effect on the optimization of a multipinhole SPECT system. The sensitivity equivalent diameter is always larger than the resolution equivalent diameter, making the system detect more photons, at an equivalent resolution, than without penetration. This difference increases with a larger pinhole opening angle making a large opening angle seem more beneficial. Similarly, the difference between sensitivity and resolution equivalent diameters increases with a smaller pinhole aperture. This phenomena tends to reward systems with highresolution detectors as they usually have a large number of pinholes with small apertures. However, the extra detected photons that penetrate the pinhole edge lie in the tail of the point spread function and it is not obvious if and how much it will improve the reconstructed image.

Designing multipinhole SPECT systems is ultimately a trade-off where many parameters need to be taken into account. There is no single optimal system, each application and context have their own special requirements. We hope the presented theory have brought new insights and tools to understand and visualize these trade-offs and that it ultimately will lead to better multipinhole SPECT systems.

\section{APPENDIX A \\ Proof That the SENSITIVITY IS MAXIMIZED WHEN THE DETECTOR IS ORTHOGONAL TO THE OPTICAL AXIS}

This section considers a slanted detector and finds the value of the angle between the optical axis and the detector normal, $\theta$, which maximizes the sensitivity in multipinhole SPECT, see Fig. 6. 
The number of pinholes and hence the sensitivity is limited by the maximum total solid angle of $4 \pi$ subtended by the images. The total sensitivity is therefore maximized when the sensitivity per solid angle is maximized for a single pinhole camera. By keeping $l, L, a$, and $d$ fixed, the sensitivity and geometric resolution, at the center, is fixed and we can choose the $\theta$ that minimizes the solid angle subtended by a single image. Thus, we have maximized sensitivity per solid angle.

The image of the spherical FOV is a conic section between the pinhole cone and the image plane. This conic section is an ellipse, the upper ellipse in Fig. 6(a). The solid angle this ellipse subtends cannot be computed analytically. Instead consider the ellipse projected onto the tangent plane of the unit sphere. This projection is also an ellipse, the lower ellipse in Fig. 6(a). The tangent plane is chosen so that the ellipse is centered around the tangent point. It is clear that minimizing this projected ellipse also minimizes the solid angle subtended by the image ellipse.

First, note that $\cot \beta=\sqrt{l^{2}-d^{2} / 4} /(d / 2)=\sqrt{4 l^{2} / d^{2}-1}$. Using this and the sine rule in the triangle $P B C$ we calculate

$$
\begin{aligned}
D_{1} & =\frac{(L-l) \sin \beta}{\sin \left(\frac{\pi}{2}-\beta-\theta\right)}=\frac{(L-l) \sin \beta}{\cos \beta \cos \theta-\sin \beta \sin \theta} \\
& =\frac{(L-l)}{\cot \beta \cos \theta-\sin \theta}=\frac{(L-l)}{\sqrt{\frac{4 l^{2}}{d^{2}}-1} \cos \theta-\sin \theta} .
\end{aligned}
$$

Using the sine rule in triangle $O B C$ we deduce that

$$
\begin{aligned}
\frac{D_{1}}{\sin \gamma_{1}} & =\frac{L}{\sin \left(\frac{\pi}{2}-\gamma_{1}-\theta\right)}=\frac{L}{\cos \gamma_{1} \cos \theta-\sin \gamma_{1} \sin \theta} \\
& \Rightarrow \frac{D_{1}}{\tan \gamma_{1}}=\frac{L}{\cos \theta-\tan \gamma_{1} \sin \theta} \\
& \Rightarrow \tan \gamma_{1}=\frac{D_{1} \cos \theta}{L+D_{1} \sin \theta} .
\end{aligned}
$$

Inserting (35) in (36) and through some algebraic manipulation we find that

$$
\tan \gamma_{1}=\frac{L-l}{L \sqrt{\frac{4 l^{2}}{d^{2}}-1}-l \tan \theta} .
$$

Similarly we obtain

$$
\tan \gamma_{2}=\frac{L-l}{L \sqrt{\frac{4 l^{2}}{d^{2}}-1}+l \tan \theta}
$$

The major axis radius of the projected ellipse on the unit sphere is equal to $\tan \gamma$ where $\gamma=\left(\gamma_{1}+\gamma_{2}\right) / 2$ which can be computed through the arc tangent of (37) and (38). To find when this radius is minimized with respect to $\theta$, we look at the derivative

$$
\begin{aligned}
\frac{\partial \gamma}{\partial \theta}= & \frac{L l^{2}(L-l) \sqrt{\frac{4 l^{2}}{d^{2}}-1} \tan \theta}{\cos ^{2} \theta} \\
& \times \frac{1}{\left(L \sqrt{\frac{4 l^{2}}{d^{2}}-1}-l \tan \theta\right)^{2}+(L-l)^{2}} \\
& \times \frac{1}{\left(L \sqrt{\frac{4 l^{2}}{d^{2}}-1}+l \tan \theta\right)^{2}+(L-l)^{2}} .
\end{aligned}
$$

Now, since $L>l>d / 2>0$ and $0 \leq \theta<\pi / 2-\beta$, the derivative in (39) is greater than zero for all $\theta>0$ and only equal to zero when $\theta=0$. This means that $\gamma$ and hence $\tan \gamma$ has a minimum when $\theta=0$.

The minor axis of the projected ellipse is orthogonal to the figure plane in Fig. 6(b) and lies at point $E$. The angle $\gamma_{1}>\gamma_{2}$ for $\theta>0$ so the minor axis lies to the right of optical axis, except for $\theta=0$ when $\gamma_{1}=\gamma_{2}$ and the minor axis crosses the optical axis. The depth of the image ellipse, i.e., into the figure plane, at $B$ is constant for all $\theta$. The width of the projected ellipse at $F$ is therefore proportional to the distance $O F$, which is the shortest when $\theta=0$. The minor axis is the widest part of the projected ellipse, so it is wider than at $F$, except when $\theta=0$, when $E$ and $F$ coincide. This means that the minor axis is at its minimum when $\theta=0$.

Both the minor and major axes of the projected ellipse are at their minimums when $\theta=0$, which means that the solid angle subtended by the image ellipse is also minimized and hence the sensitivity per solid angle is maximized.

\section{APPENDIX B \\ SENSITIVITY VARIATIONS OVER THE FIELD-OF-VIEW}

A single pinhole's sensitivity at a point is proportional to the solid angle subtended by the pinhole. This solid angle is proportional to

$$
\frac{\cos \theta_{p}}{l_{p}^{2}}
$$

where $\theta_{p}$ is the angle between the pinhole normal and the line from the source to the pinhole and $l_{p}$ is the distance to the pinhole [27]. To investigate the sensitivity variations over the FOV, the sensitivity at a large number of points in the FOV was computed. The points were sampled from the surface, as well as from three orthogonal central cross-sections of the spherical FOV. The surface was evaluated at a $100 \times 200$ grid on the polar and azimuthal angles of the spherical coordinates. The cross-sections were evaluated at $100 \times 100$ square grids, disregarding the points outside the FOV. The sensitivity variation is defined as $1-\min _{i}\left(S_{i}\right) / \max _{i}\left(S_{i}\right)$, where $S_{i}$ is the sensitivity at point $i$. Note that the sensitivity variation is independent of the aperture diameter.

Systems with 24, 124, and 1592 pinholes were investigated. The best packing of equal circles on a sphere, according to [26], was used for each configuration. The sensitivity variations were also computed for different collimator positions. For each given collimator position and circle packing the position of the detector, $L$, was computed. The collimator positions were chosen such that the distance to the detector is within reason. For example, in the case of 24 pinholes, moving the collimator beyond $13 \mathrm{~mm}$ will quickly move the detector towards infinity. Table IV lists the computed systems. The sensitivity variations are the highest for systems with a small number of pinholes. The variations are also higher for systems with the collimator close to the FOV. The main conclusion is, however, that the sensitivity 
TABLE IV

COMPUTED SENSITIVITY VARIATIONS OVER THE FOV FOR MULTIPINHOLE SPECT SySTEMS. A FOV DiAMETER OF 10 MM WAS USED IN ALL CASES

\begin{tabular}{cccc}
\hline $\begin{array}{c}\text { Num. of pinholes } \\
n\end{array}$ & $\begin{array}{c}\text { Coll. radius } \\
l(\mathrm{~mm})\end{array}$ & $\begin{array}{c}\text { Det. radius } \\
L(\mathrm{~mm})\end{array}$ & $\begin{array}{c}\text { Sens. var. } \\
1-\frac{\min _{i}\left(S_{i}\right)}{\max _{i}\left(S_{i}\right)}\end{array}$ \\
\hline 24 & 11 & 51.3 & 0.0167231 \\
24 & 12 & 95.7 & 0.0107612 \\
24 & 13 & 343.3 & 0.0074630 \\
124 & 13 & 21.7 & 0.0027930 \\
1592 & 13 & 14.6 & 0.0002429 \\
124 & 25 & 137.1 & 0.0003728 \\
1592 & 25 & 32.4 & 0.0000048 \\
\hline
\end{tabular}

variations are small for all the listed systems. Even systems with as few as 24 pinholes show variations below $2 \%$.

To get an indication to the sensitivity variations for systems with cylindrical symmetries we computed the sensitivities, while excluding the pinholes outside the solid angle subtended by the cylinder's curved surface. For a cylinder with an opening angle of $25^{\circ}$ and with $l=13 \mathrm{~mm}$, the sensitivity variations were $11.4 \%$ and $3.2 \%$ for 124 (102 within the cylinder) and 1592 (1411 within the cylinder) pinholes, respectively. At $l=25$ the variations for the same number of pinholes were $7.7 \%$ and $2.1 \%$. This crude way of excluding pinholes outside the cylinder leads to suboptimal circle packings for the cylinder. Even so the sensitivity variations are relatively small, especially in the case when the collimator is placed $25 \mathrm{~mm}$ away from the center of the FOV.

The sensitivity variations decrease with an increasing number of pinholes. The following paragraphs derive the sensitivity variations in the limit, when the number of pinholes grows toward infinity. Consider a point on the $z$-axis at a distance $p$ from the center of the FOV. Also, consider a pinhole on the collimator sphere at position $(\phi, \psi)$, where $\phi$ is the polar angle and $\psi$ the azimuthal angle. Then

$$
\cos \theta_{p}=\frac{l-p \cos \phi}{l_{p}}
$$

and

$$
\begin{aligned}
l_{p} & =\sqrt{p^{2} \sin ^{2} \phi+(l-p \cos \phi)^{2}} \\
& =\sqrt{p^{2}+l^{2}+2 p l \cos \phi} .
\end{aligned}
$$

Equations (41) and (42) holds for all polar angles $\phi \in[0, \pi]$.

Now, if there is an infinite number of pinholes evenly distributed on the collimator sphere, the ratio of sensitivities at the point relative to the center point is

$$
\begin{aligned}
\frac{S(p)}{S(0)} & =\frac{l^{2}}{4 \pi} \int_{0}^{2 \pi} \int_{0}^{\pi} \frac{\cos \theta_{p}}{l_{p}^{2}} \sin \phi d \psi d \phi \\
& =\frac{l^{2}}{2} \int_{0}^{\pi} \frac{l-p \cos \phi}{\left(p^{2}+l^{2}-2 p l \cos \phi\right)^{3 / 2}} \sin \phi d \phi
\end{aligned}
$$

Substituting $t=\cos \phi$ we obtain $d t=-\sin \phi d \phi$. Swapping the limits and using integration by parts results in

$$
\begin{aligned}
\frac{S(p)}{S(0)}= & \frac{l^{2}}{2} \int_{-1}^{1} \frac{l-p t}{\left(p^{2}+l^{2}-2 p l t\right)^{3 / 2}} d t \\
= & {\left[\frac{l^{2}(l-p t)}{2 p l\left(p^{2}+l^{2}-2 p l t\right)^{1 / 2}}\right]_{-1}^{1} } \\
& -\int_{-1}^{1} \frac{-l}{2\left(p^{2}+l^{2}-2 p l t\right)^{1 / 2}} d t \\
= & \frac{l}{2 p}\left(\frac{l-p}{\left(p^{2}+l^{2}-2 p l\right)^{1 / 2}}-\frac{l+p}{\left(p^{2}+l^{2}+2 p l\right)^{1 / 2}}\right) \\
& -\left[\frac{1}{2 p}\left(p^{2}+l^{2}-2 p l t\right)^{1 / 2}\right]_{-1}^{1} \\
= & \frac{l}{2 p}(1-1)-\frac{1}{2 p}((l-p)-(l+p))=1 .
\end{aligned}
$$

In other words, the sensitivity in this case is constant over the entire FOV.

\section{REFERENCES}

[1] J. Palmer and P. Wollmer, "Pinhole emission computed tomography: method and experimental evaluation," Phys. Med. Biol., vol. 35, no. 3, pp. 339-50, Mar. 1990.

[2] D. A. Weber, M. Ivanovic, D. Franceschi, S. E. Strand, K. Erlandsson, M. Franceschi, H. L. Atkins, J. A. Coderre, H. Susskind, and T. Button, "Pinhole SPECT: An approach to in vivo high resolution SPECT imaging in small laboratory animals," J. Nucl. Med., vol. 35, no. 2, pp. 342-8, Feb. 1994.

[3] R. J. Jaszczak, J. Li, H. Wang, M. R. Zalutsky, and R. E. Coleman, "Pinhole collimation for ultra-high-resolution, small-field-of-view SPECT," Phys. Med. Biol., vol. 39, no. 3, pp. 425-37, Mar. 1994.

[4] R. Rowe, J. Aarsvold, H. Barrett, J.-C. Chen, W. Klein, B. Moore, I. Pang, D. Patton, and T. White, "A stationary hemispherical SPECT imager for three-dimensional brain imaging," J. Nucl. Med., vol. 34, no. 3, pp. 474-480, Mar. 1993

[5] M. Rogulski, H. Barber, H. Barrett, R. Shoemaker, and J. Woolfenden, "Ultra-high-resolution brain SPECT imaging: Simulation results," IEEE Trans. Nucl. Sci., vol. 40, no. 4, pp. 1123-1129, Aug. 1993.

[6] K. Ishizu, T. Mukai, Y. Yonekura, M. Pagani, T. Fujita, Y. Magata, S. Nishizawa, N. Tamaki, H. Shibasaki, and J. Konishi, "Ultra-high resolution SPECT system using four pinhole collimators for small animal studies," J. Nucl. Med., vol. 36, no. 12, pp. 2282-7, Dec. 1995.

[7] D. McElroy, L. MacDonald, F. Beekman, Y. Wang, B. Patt, J. Iwanczyk, B. Tsui, and E. Hoffman, "Performance evaluation of A-SPECT: A high resolution desktop pinhole SPECT system for imaging small animals," IEEE Trans. Nucl. Sci., vol. 49, no. 5, pp. 2139-2147, Jan. 2002

[8] S. Meikle, P. Kench, A. Weisenberger, R. Wojcik, M. Smith, S. Majewski, S. Eberl, R. Fulton, A. Rosenfeld, and M. Fulham, "A prototype coded aperture detector for small animal SPECT," IEEE Trans. Nucl. Sci., vol. 49, no. 5, pp. 2167-2171, Jan. 2002.

[9] N. Schramm, G. Ebel, U. Engeland, T. Schurrat, M. Behe, and T. Behr, "High-resolution SPECT using multipinhole collimation," IEEE Trans. Nucl. Sci., vol. 50, no. 3, pp. 315-320, Jan. 2003.

[10] L. Furenlid, D. Wilson, Y. Chen, H. Kim, P. Pietraski, M. Crawford, and H. Barrett, "FastSPECT II: A second-generation high-resolution dynamic SPECT imager," IEEE Trans. Nucl. Sci., vol. 51, no. 3, pp. 631-635, Jan. 2004.

[11] A. L. Goertzen, D. W. Jones, J. Seidel, K. Li, and M. V. Green, "First results from the high-resolution mouseSPECT annular scintillation camera," IEEE Trans. Med. Imag., vol. 24, no. 7, pp. 863-7, Jul. 2005.

[12] F. J. Beekman, F. van der Have, B. Vastenhouw, A. J. A. van der Linden, P. P. van Rijk, J. P. H. Burbach, and M. P. Smidt, "U-SPECT-I: A novel system for submillimeter-resolution tomography with radiolabeled molecules in mice," J. Nucl. Med., vol. 46, no. 7, pp. 1194-200, Jul. 2005 . 
[13] H. Kim, L. Furenlid, M. Crawford, D. Wilson, H. Barber, T. Peterson, W. Hunter, Z. Liu, J. Woolfenden, and H. Barrett, "SemiSPECT: A small-animal single-photon emission computed tomography (SPECT) imager based on eight cadmium zinc telluride (CZT) detector arrays," Med. Phys., vol. 33, no. 2, pp. 465-474, Jan. 2006.

[14] J. Y. Hesterman, M. A. Kupinski, L. R. Furenlid, D. W. Wilson, and H. H. Barrett, "The multi-module, multi-resolution system (M3R): A novel small-animal SPECT system," Med. Phys., vol. 34, no. 3, pp. 987-93, Mar. 2007.

[15] F. J. Beekman and B. Vastenhouw, "Design and simulation of a highresolution stationary SPECT system for small animals," Phys. Med. Biol., vol. 49, no. 19, pp. 4579-92, Oct. 2004.

[16] T. Funk, P. Després, W. C. Barber, K. S. Shah, and B. H. Hasegawa, "A multipinhole small animal SPECT system with submillimeter spatial resolution," Med. Phys., vol. 33, no. 5, pp. 1259-68, May 2006, notes.

[17] L. Meng, N. Clinthorne, S. Skinner, R. Hay, and M. Gross, "Design and feasibility study of a single photon emission microscope system for small animal i-125 imaging," IEEE Trans. Nucl. Sci., vol. 53, no. 3, pp. 1168-1178, Jan. 2006.

[18] Z. Cao, G. Bal, R. Accorsi, and P. D. Acton, "Optimal number of pinholes in multi-pinhole SPECT for mouse brain imaging - A simulation study," Phys. Med. Biol., vol. 50, no. 19, pp. 4609-24, Oct. 2005.

[19] T. Song, Y. Choi, Y. Chung, J. Jung, Y. Choe, K. Lee, S. Kim, and B. Kim, "Optimization of pinhole collimator for small animal SPECT using monte carlo simulation," IEEE Trans. Nucl. Sci., vol. 50, no. 3, pp. 327-332, Jan. 2003.
[20] M. C. M. Rentmeester, F. van der Have, and F. J. Beekman, "Optimizing multi-pinhole SPECT geometries using an analytical model," Phys. Med. Biol., vol. 52, no. 9, pp. 2567-81, May 2007.

[21] P. Nillius and M. Danielsson, "Theoretical bounds and optimal configurations for multi-pinhole SPECT," in IEEE Nucl. Sci. Symp. Conf. Rec. (NSS/MIC'08), Oct. 2008, pp. 5020-5022.

[22] D. A. Kottwitz, "The densest packing of equal circles on a sphere," Acta Crystallogr. A, vol. 47, pp. 158-165, Jan. 1991.

[23] S. D. Metzler, J. E. Bowsher, M. F. Smith, and R. J. Jaszczak, "Analytic determination of pinhole collimator sensitivity with penetration," IEEE Trans. Med. Imag., vol. 20, no. 8, pp. 730-41, Aug. 2001.

[24] R. Accorsi and S. D. Metzler, "Analytic determination of the resolution-equivalent effective diameter of a pinhole collimator," IEEE Trans. Med. Imag., vol. 23, no. 6, pp. 750-63, Jun. 2004.

[25] M. C. Goorden, M. C. M. Rentmeester, and F. J. Beekman, "Theoretical analysis of full-ring multi-pinhole brain SPECT," Phys. Med. Biol., vol. 54, no. 21, pp. 6593-6610, Nov. 2009.

[26] N. J. A. Sloane, Table of sphericalcodes 2000 [Online]. Available: http://www.research.att.com/ njas/packings

[27] H. H. Barrett and W. Swindell, Radiological Imaging: The Theory of Image Formation, Detection and Processing. . New York: Academic, 1981, vol. 1. 